\title{
Analysis of Flow Field for Link Rod Butterfly Valve
}

\author{
Xuedong $\mathrm{Ma}^{1, \text { a }}$, Jie Teng ${ }^{2, b}$ \\ ${ }^{1}$ School of Mechanical Engineering and Automation ,University of Science and Technology \\ Liaoning Liaoning, Anshan ,Liaoning Province,114051, China \\ ${ }^{2}$ School of Mechanical Engineering and Automation ,University of Science and Technology \\ Liaoning Liaoning, Anshan ,Liaoning Province,114051, China \\ amadong912@126.com , btengjie654321@163.com
}

Keywords: Link rod butterfly valve, Flow field, Finite element

\begin{abstract}
The link rod butterfly valve relies on the link mechanism inside the flow channel to realize opening. The flow within flow channel is complex and the flow state is uncertain, so that pressure load of the flow part can't be determined in the butterfly valve designing, and strength of the parts can't be calculated accurately and the thrust of the hydraulic cylinder can't be determined accurately. Therefore, it is necessary to carry out flow field analysis. In this paper, by adopting the plug-in COSMOSMotion of Solidworks software, the link rod butterfly valve with DN1800 is carried on motion simulation, determining the specific opening. And then the butterfly valve at different opening is carried out the finite element analysis of the flow field, adopting the plug-in COSMOSFloworks of Solidworks of software. The result shows that: the force is the largest when the valve plate is in just opening, it is $230731 \mathrm{~N}$, the strength analysis of the flow part and selection of the hydraulic cylinder should be based on the working condition; When full opening, the upward force on the valve plate is $18504 \mathrm{~N}$, this force is the power source when the valve plate is over opening. The above mentioned work provides a reliable theoretical basis for the strength calculation and the force and energy parameters calculation of the link rod butterfly valve and the theoretical reference for flow pattern evaluation of the link rod butterfly valve.
\end{abstract}

\section{Introduction}

The another name of the link rod butterfly valve is three-rod valve being widely used in iron-smelting hot blast stove system, and to close the valve mainly relies on the action of a group of four bar linkage mechanism inside flow channel to realize, therefore, compared to the other butterfly valves, the flow within the flow channel is very complex. According to the relevant retrieval, the quantitative analysis of the flow channel flow has not been reported [1-5]. The uncertainty flow state of the medium causes the uncertainty of flow components' pressure load in designing, and then the driving torque can not be ensured, leading to strength of the parts can not be calculated accurately. Therefore, it impacts structure optimization and innovation design of the link rod butterfly valve.

In this article, the flow field is simulated by the COSMOSFloworks software when the link butterfly valve is in the different opening. It is concluded the changes such as the pressure of the valve plate, the pressure difference, and the force, providing the basic theory data for design and optimization of the link rod butterfly valve. 


\section{The working principle and the trace analysis of the link rod butterfly valve}

In this paper, the connecting rod butterfly valve is analyzed with the model DN1800.Working pressure is $0.4 \mathrm{MPa}$ and working medium is $500^{\circ} \mathrm{C}$ hot air. Average flow speed for the hot air is $20 \mathrm{~m} / \mathrm{s}$.

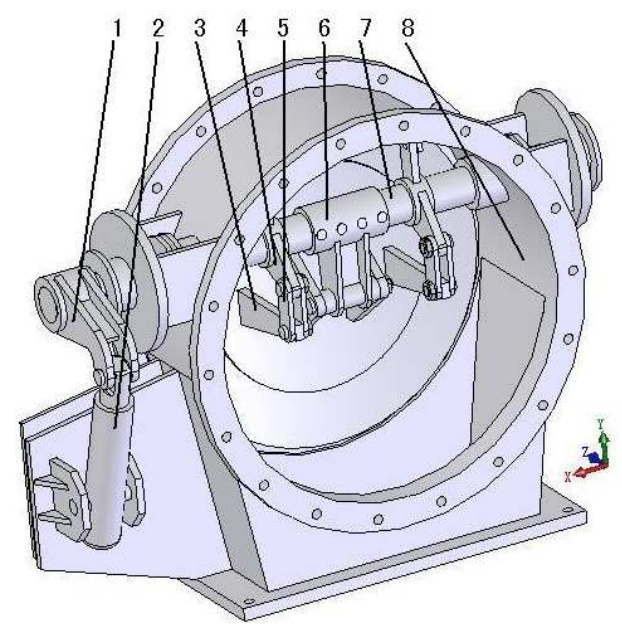

Fig. 1 Working principle diagram

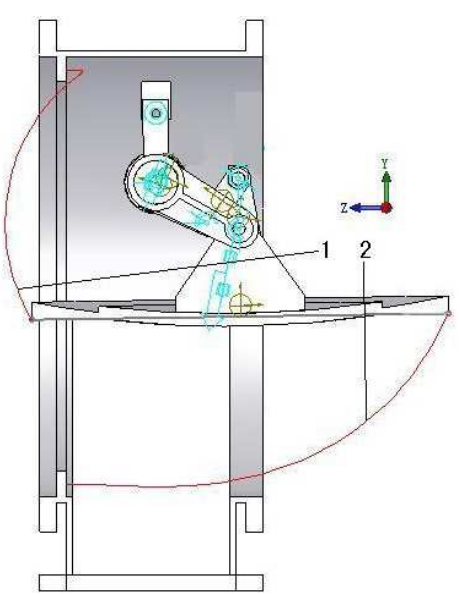

Fig. 2 Motion trace

The transmission mechanism of this link rod butterfly valve is four-bar mechanism. Each bar is defined respectively as driving lever, fixed rod (frame), link rod (valve plate) jointed arm. It is shown in Figure 1 (where, 1 the crank, 2 hydraulic cylinder, 3 link rod (valve plate), 4 fixed rod, 5 jointed arm , 6 driving lever ,7 spindle 8 valve body). In Figure 1, one end of the driving lever is linked with the main spindle through the fixed pin, The other end is connected to the valve plate (link rod). The fixed rod is consolidated with the valve body through the main spindle, and plays the role of fixing support in the whole motion process of valve plate. One end of the jointed arm is connected with the fixed rod, the other end is connected with the valve plate, and the jointed arm plays the role of achieving certain path in the motion process of the valve plate. The power source of the link rod butterfly valve is the hydraulic cylinder that is hinged with the crank through the pin shaft, and the crank is consolidated with the main spindle through the key.

In order to carry out the effective partition for the different opening angles in the fluid analysis, we need to understand fully the motion of the mechanism, the link rod butterfly valve is carried out motion simulation adopting the plugin COSMOSMtion of Solidworks software, it can be concluded the motion trace for upper and lower part of the valve plate through the trajectory tracking, it is shown in figure 2. From figure 2, upper part of the valve plate firstly produces a linear motion far away from the sealing face of the valve body(direction Z), and then draws a period of circular arc, the same as lower part of the valve plate, and the curve is consistent with the pertinent literature[6]. In the motion process of the valve plate, the valve plate and the valve body do not produce interference, meanwhile, the line segment of the trace makes the valve plate and the valve body disengage quickly, and makes the sealing faces no friction relatively, ensuring the using life of the valve sealing faces.

\section{Building 3D model and determining the calculate space}

Building 3D model. Solidworks software is used to build 3D parts model through stretching and rotating commands. In 3D model, not only building parts such as the valve plate, the fixed rod, and the link rod, but also building duct that both ends are closed to determine the fluid space. After 
completing parts design, all the parts are assembled, then the valve plate is opened to the certain position, so it can be formed an assembled body with the certain closed cavity, it is shown in figure 3.

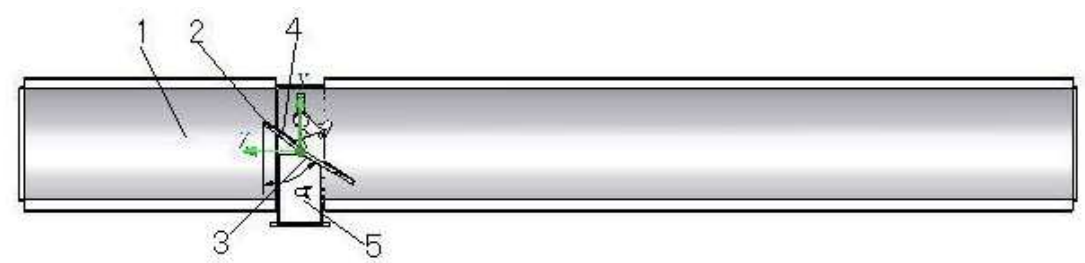

1 cavity flow space (calculating space); 2 the valve plate; 3 the front of valve plate 4 the back of valve plate; 5 the opening

Fig. 3 Calculating space

Determining calculating space. Executing the checking command of geometry space of COSMOSFloworks software, it will automatically calculate the flow cavity volume and entity volume. If the flow cavity volume is checked as zero, it explains that the flow space is not closed, then it needs to be modified and checked the model until the cavity is closed [7]. The closed flow space is the calculating space (see Fig. 3).

\section{Building analysis project of COSMOSFloworks and setting the boundary condition}

Material attribute setting and initial condition setting about the hot air depend on the analysis guide of COSMOSFloworks.

Setting the analysis project of COSMOSFloworks. Entering the analysis guide of COSMOSFloworks, the following projects are defined in turn:

(1) Creating analysis name; (2) Defining unit system, this article adopts mm-g-s system; (3) Defining analysis type is internal flow, and ignoring cavity influence; (4) Determining fluid, in this article the medium is hot air, and the specific physical parameters such as density, viscosity come from the related reference[8]; (5)Setting wall conditions, and setting roughness, according to the relevant literature to determine $R_{d}=10 \mu m$ and setting wall adiabatic; (6) Determining the initial condition, according to the actual working condition of the link rod butterfly valve, the initial pressure is determined for $0.4 \mathrm{MPa}$, initial temperature is $773.2 \mathrm{~K}$; (7) Setting the resolution is 10mm; (8) Completing setting.

Setting the boundary condition. According to the practice of the engineering, the boundary condition of the entrance is the velocity boundary condition. It is set for $20000 \mathrm{~mm} / \mathrm{s}$. And the boundary condition of the export is pressure boundary condition. It is set for $0.4 \mathrm{MPa}$.

\section{Results and discussions}

Taking the valve plate opening $50^{\circ}$ for example (Fig.3), and running simulation, COSMOSFloworks software will mesh automatically, and get the calculating results.

Figure 4 is pressure distribution of the section. From Figure 4, the pressure of the near valve plate is higher and the pressure of the valve plate is reduced because flow is hindered. The pressure near bar system spindle is lower because of the cover effect of the valve plate. 
Figure 5 is the velocity distribution of the section. From Figure 5, the velocity in the aperture of valve plate opening is higher, meanwhile, because of the bar system keeping out, the upper velocity is lower than the below velocity after the valve. Due to the influence of the bar system, the streamline between the stem and the back of valve plate is disorder and is in cross shape, and local vortex appears in the back of the valve near stem and the lower valve body.

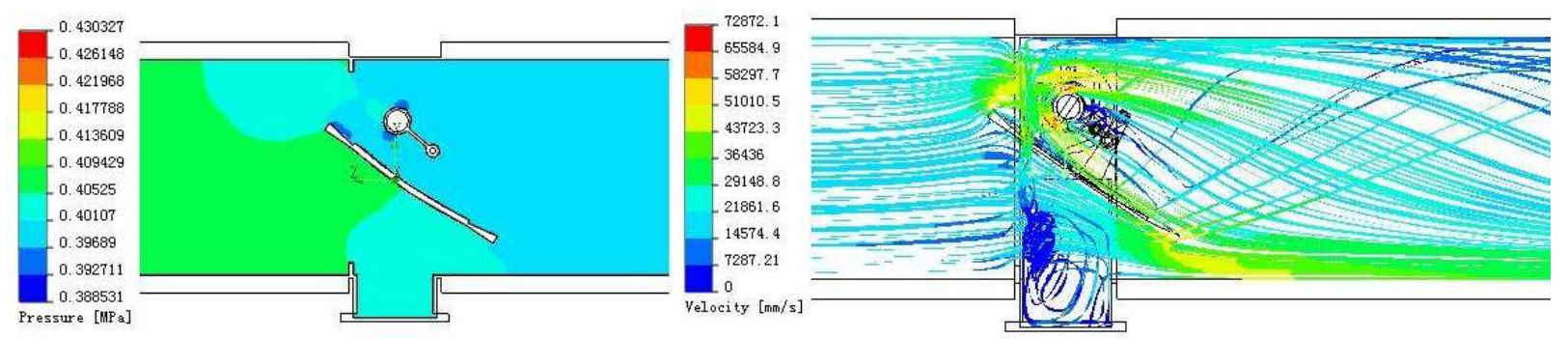

Fig. 4 Pressure distribution Fig. 5 Velocity distribution

In the flow parts, compared with the bar system, the area of the valve plate is largest and its stress is largest, therefore, the following analysis takes the valve plate as the research object.

According to the trajectory tracking figure (shown in figure 2), determining the opening condition of the valve plate is seven groups, which a line segment is 1 group and arc segments are six groups, this grouping basically describes the whole flow condition of the valve plate opening process. The average pressure and pressure difference of the front and back of the valve plate the valve plate in different opening, it is shown in figure 6 . In the figure 6 , when the valve plate opens, the average bearing pressure in the front of the valve plate is the largest, reaching $0.55 \mathrm{MPa}$, with the increasing of the opening, the average pressure gradually decreases, but existing a turning point at $4^{\circ}$. When the valve plate is in straight line trip, the pressure loss is far greater than the arc trip, and is 4 times.

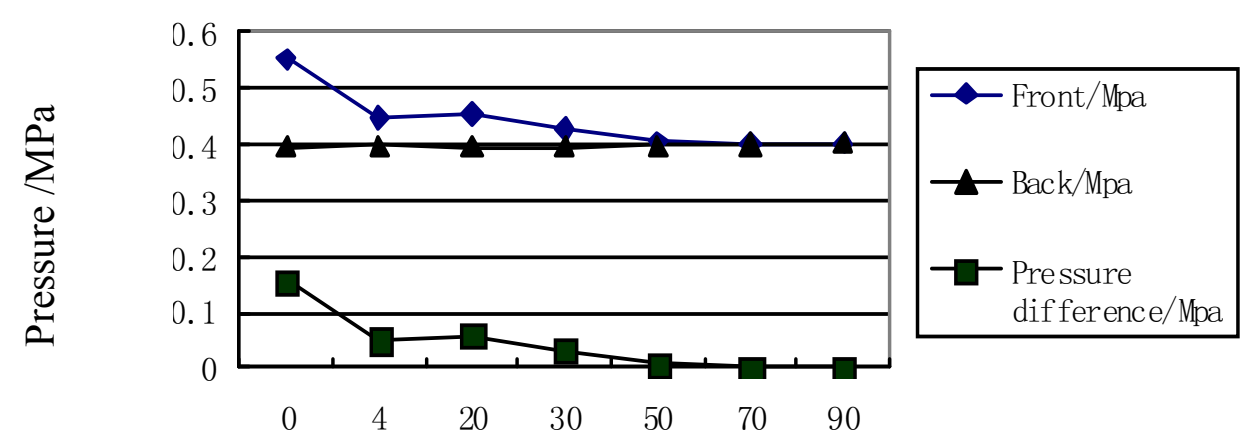

Opening $/ \circ$

Fig. 6 Intensity of pressure along with opening change

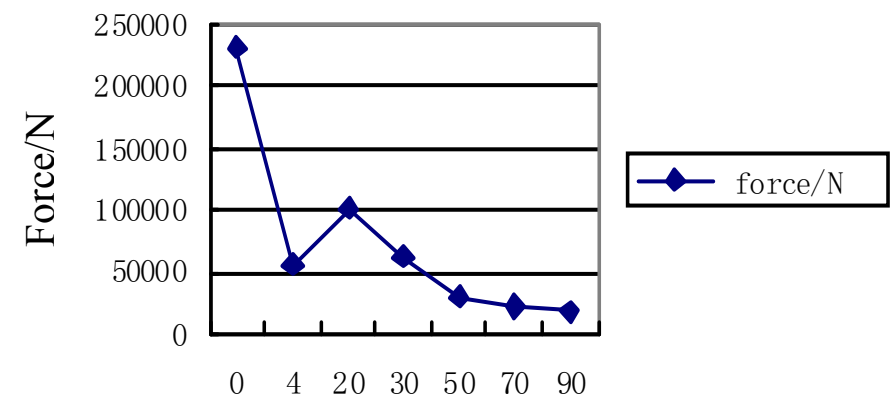

Opening $/{ }^{\circ}$

Fig. 7 Normal force of valve plate 
The normal force of the valve plate is shown in figure 7. From figure 7, the force is the largest when the valve plate is the straight line trip, it is $230731 \mathrm{~N}$. Therefore, when the link rod butterfly valve is designed, the calculation of the maximum driving torque and the intensity check of the parts should be carried on according to the force of the straight line trip. In the actual using process, this kind of butterfly valve is in full opening, existing over open (opening $>90^{\circ}$ ) phenomenon. From figure7, when it is full opening (opening $=90^{\circ}$ ), compared with just opening, although the force of the front and back of the valve plate is small, but there is still the upward force, it is $18504 \mathrm{~N}$, and this force is the power source that results in the link rod butterfly valve over open.

\section{Conclusion}

(1) According to the motion trace, the data such as the average pressure, average pressure difference, and force for the valve plate are extracted when it is in different opening. It basically describes the whole process of fluid flow in its open process.

(2) According to the analysis of seven group conditions, it can be known the specific numerical values about the average pressure, average pressure difference, and force for the front and back of the valve plate at different opening, providing relatively reliable data for strength calculation, force and energy parameter calculation and the selection calculation of the hydraulic cylinder.

(3) Analyzing the reason that the valve plate is over open in full opening, it provides theoretical reference for the structure improvement of this kind of butterfly valve.

\section{Reference}

[1] Yanjie Zhang, Application of three-lever type butterfly valve in metallurgical iron-smelting equipment, Valve, 2005.(3):32-35.

[2] Lei Yao, Wanqing Wang, Jianmin Qi, The research and development of the connection rod butterfly valve, Metallurgical Information Review, 2007.(6):63-64.

[3] Jianmin Qi, Analysis structure about the link lever butter valve, Metallurgical Equipment, 2008.168(2):78 80.

[4] Xinming Yuan,Genhai Mao, The numerical simulation of flow field and resistance Characteristics of Valve Pipe, Journal of Hydroelectric Engineering, 1999(4) :60-65.

[5] Guoquan Huang, Zhongwu Cao, The research on numerical simulation of centric type butterfly valve flow field, 2011(7):186-188.

[6] Yanjun Guo, The structure compare and application of there-level type shut-off valve, Valve, 2003 (3):16-17.

[7] Jingmin Huang, Yili Lin, SolidWorks2009 Senior Design, Beijing, tsinghua university press, pp 450- 462

[8] Guangsheng Du, Engineering Fluid Mechanics, China power press, Beijing 2006, pp120 127. 